\title{
Tumor suppressor Pdcd4 attenuates delta Np73 translation in human breast cancer cells
}

\author{
Noriko Yoshikawa ${ }^{1}$, Eriko Kinoshita ${ }^{1}$, Mayuko Hatai ${ }^{1}$, Satomi Kagota ${ }^{2}$, Kazumasa Shinozuka ${ }^{2}$, \\ Kazuki Nakamura ${ }^{1}$
}

${ }^{I}$ Department of Pharmacology I, School of Pharmaceutical Sciences, Mukogawa Women's University, Japan, ${ }^{2}$ Department of Pharmacology II, School of Pharmaceutical Sciences, Mukogawa Women's University, Japan

[Background] Programmed cell death $4(\mathrm{Pdcd} 4)$ functions as a transformation suppressor, interacts with translation initiation factor eIF4A to inhibit translation in an mRNA-specific manner, and consequently blocks pro-oncogenic events. The loss of Pdcd4 has been shown to cause gene-specific changes in transcription. Although the tumor suppressor Pdcd4 acts directly on the translation initiation complex, few translational targets of Pdcd4 have been identified. For the discovery of translational targets of Pdcd4, we used T47D human non-invasive breast cancer cells with knockdown of Pdcd4 and MDA-MB-231 human invasive breast cancer cells with overexpression of Pdcd4.

[Methods] T47D cells were transfected with siRNA specifically designed to silence the Pdcd4 gene or a nonsilencing control using DharmaFECT 1 as a transfection reagent. MDA-MB-231 cells were stably transfected with the T-Rex tet regulating vector expressing the human Pdcd4 gene and treated with doxycycline. Translationally active polyribosomebound mRNA species were fractionated by a sucrose gradient, analyzed for upregulation by microarray profiling, and confirmed by qRT-PCR and Western blot analyses.

[Results] Knockdown of Pdcd4 in T47D cells identified delta Np73 mRNA as the translational target of Pdcd4. The delta Np73 mRNA shifted from inactive monosomes to actively translating polysomes. As well as the ectopic expression of Pdcd4 in MDA-MB-231, delta Np73 was identified as a target. Furthermore, the delta Np73 protein level was increased with Pdcd4 knockdown in T47D cells and decreased with Pdcd4 induction in MDA-MB-231 cells.

[Conclusions] We demonstrated that the tumor suppressor Pdcd4 attenuates the translation of delta Np73 mRNA. Delta $\mathrm{Np} 73$, an isoform of p73 lacking the NH2-terminal transactivation domain, plays an oncogenic role by interfering with the transcriptional activity of tumor suppressors p53 and TA (full-length transactivating isoform) p73.

Therapeutic intervention strategies might be directed toward inhibiting signaling by Pdcd4 target mRNAs or stabilizing Pdcd4, with the consequent inhibition of target mRNA translation. 